\title{
Challenges in Microsurgical Free Tissue Transfer for a 1-Year-Old Child
}

\author{
Hardeep Singh (1), Rakesh Kumar Khazanchi (D), Sanjay Mahendru (D), Ankit Jain (D) \\ Department of Plastic, Reconstructive and Aesthetic Surgery, Medanta - The Medicity, Gurgaon, India
}

\begin{abstract}
Challenges faced during complex reconstructions in the pediatric age group are rarely discussed in literature. This study is to share our experience in a 1-year-old patient. The patient had an exposed knee joint following trivial trauma, which was resurfaced with a free latissimus dorsi flap. On the patient's 5th day in the ward, the flap was discolored and inadequate perfusion was noted. The patient also had loose stools overnight. Before she was re-examined, the dressings were loosened and the patient was kept warm and was started on intravenous fluids. When examined in the operating room, the vascularity of the flap had improved without any intervention. The monitoring skin paddle necrosed while the muscle survived. The patient then underwent skin grafting and had uneventful recovery. The postoperative monitoring in children is critical as they have poor reserves and are prone to complications. We believe pediatric microsurgery should be done in specialized microsurgical centers that also have good intensive care units.
\end{abstract}

Keywords: Microsurgery; Debridement; Vascularity; Necrosis

\section{Introduction}

Microsurgery has an established role in the reconstruction of both adults and pediatric population [1]. Although both age groups show the same success rates in microsurgical reconstruction, there is much less literature on the specific challenges faced during pediatric reconstruction. In particular, microvascular reconstruction in children less than 2 years of age is technically more demanding [2]. This paper aims to discuss some of the challenges faced during free tissue transfer in a 1-year-old child and the methods adopted for successful reconstruction. Informed written consent for the imaging records was obtained from the patient's parents for use in the journal.

\section{Case}

We were presented with an $8 \mathrm{~kg}$ female child 1 year of age. She had developed septic arthritis of the left knee joint after a trivial injury at home, which had been debrided once at another hospital. At the time of presentation to our hospital, the patient had necrosis of the skin over the left knee joint with seropurulent discharge (Fig. 1). She underwent debridement of the wound and two sittings of negative pressure wound therapy. The upper third of the tibia, femoral condyle, patella and knee joint were exposed (Fig. 2). The patellar tendon was necrosed and hence was debrided initially. The wound measured $8 \times 9 \mathrm{~cm}$.

We decided on free tissue transfer with a latissimus dorsi musculocutaneous flap. The plan was to construct the arterial anastomosis with the descending genicular ar-

Case Report
Received: April 2, 2020
Revised: June 4, 2020
Accepted: June 12, 2020
Corresponding author:
Hardeep Singh, M.Ch.
Department of Plastic, Reconstructive and
Aesthetic Surgery, Medanta - The Medicity,
Sector 38, Gurgaon, NCR 122001, India
Tel: +91-7838156609
Fax: +91-124 4834111
E-mail: drhardeepaulakh@gmail.com

This is an Open Access article distributed under the terms of the Creative Commons Attribution Non-Commercial License (https://creativecommons.org/licenses/by-nc/4.0/) which permits unrestricted non-commercial use, distribution, and reproduction in any medium, provided the original work is properly cited.

C 2020 Korean Wound Management Society 


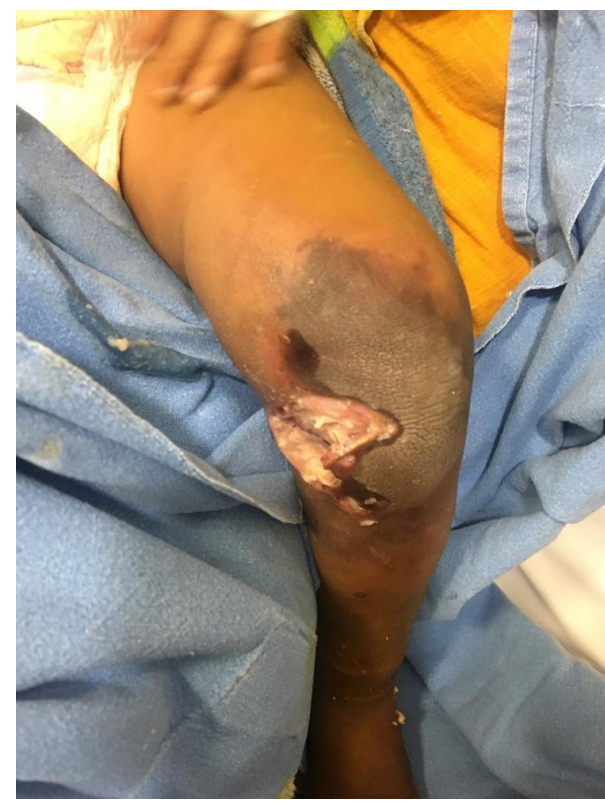

Fig. 1. Wound on presentation.

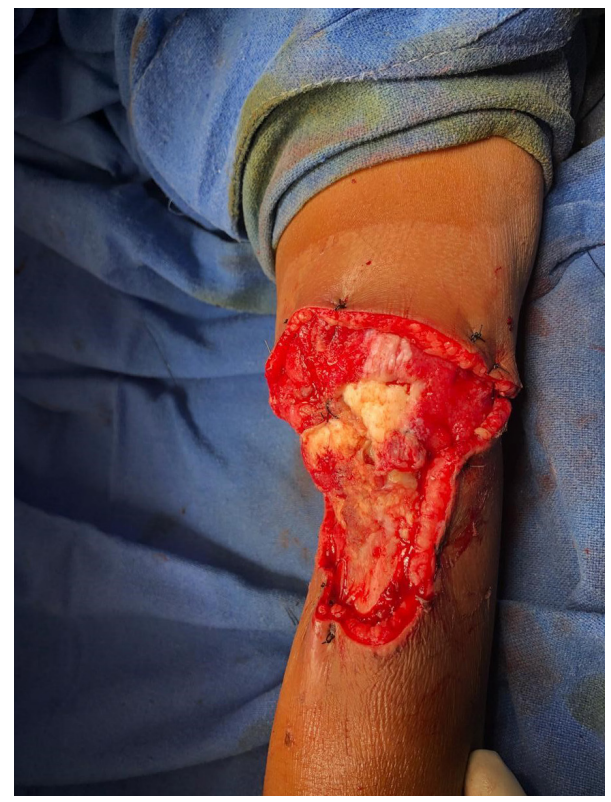

Fig. 2. Wound after debridement.

tery and venous anastomosis with the long saphenous vein. A curved medial incision on the distal thigh was made to expose the artery and vein in question. The latissimus dorsi muscle was harvested in the classical method with a skin island for monitoring (Fig. 3). The donor artery was $1 \mathrm{~mm}$ in diameter. On division of the descending genicular artery, the flow was found to be poor and hence the arterial anastomosis was performed end to side to the femoral artery. Venous anastomosis

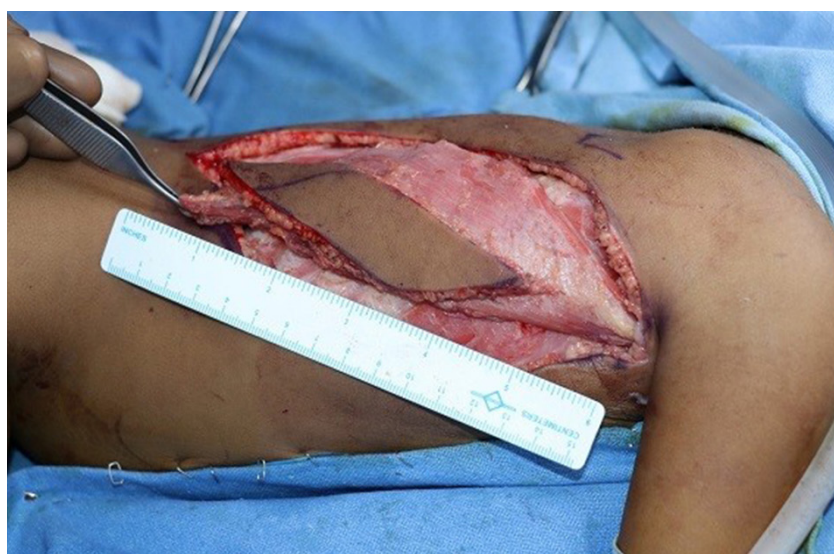

Fig. 3. Harvested latissimus dorsi flap.

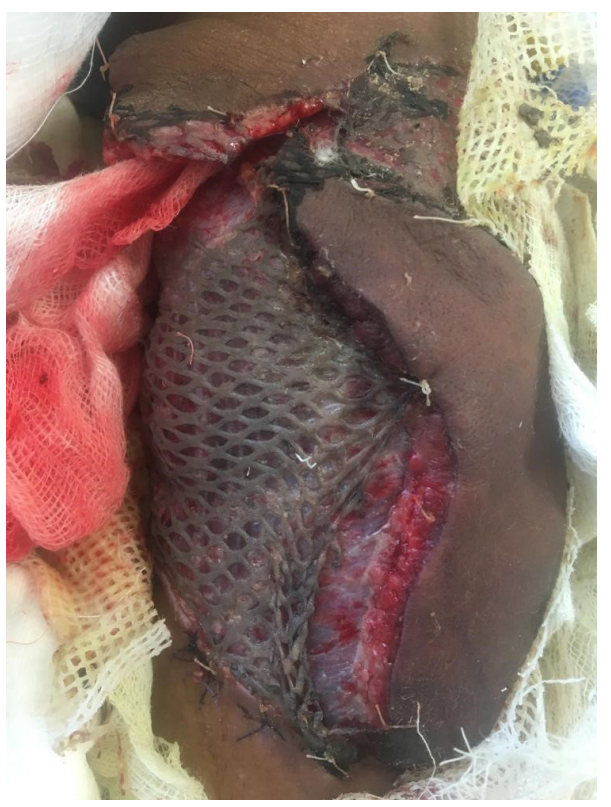

Fig. 4. Discolored, ischemic flap.

was done as planned, and the muscle was covered with skin graft.

Postoperative monitoring was critical and the patient was placed in the pediatric intensive care unit (PICU) before moving to the pediatric ward on postoperative day 2 , where she was started on oral feeds. On postoperative day 5 it was found that the flap was discolored and its perfusion was inadequate (Fig. 4). The baby had loose stools since the previous night and also had reduced urine output. She was taken up for urgent exploration. Meanwhile, at bedside the dressings had been loosened, intravenous fluids were started and the patient was kept warm. In the operating room it was found that the flap vascularity had improved and hence no additional procedure 


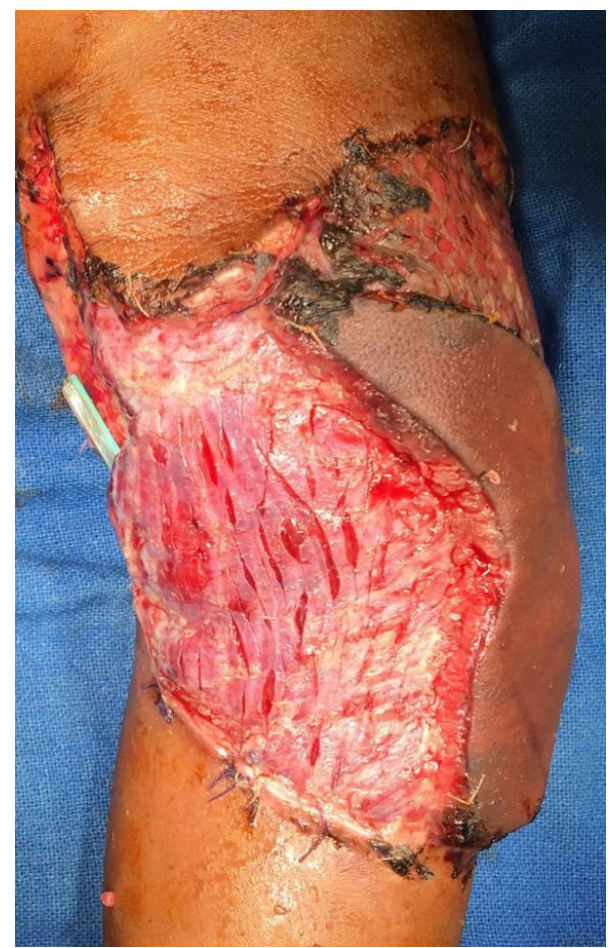

Fig. 5. Improved vascularity of flap.

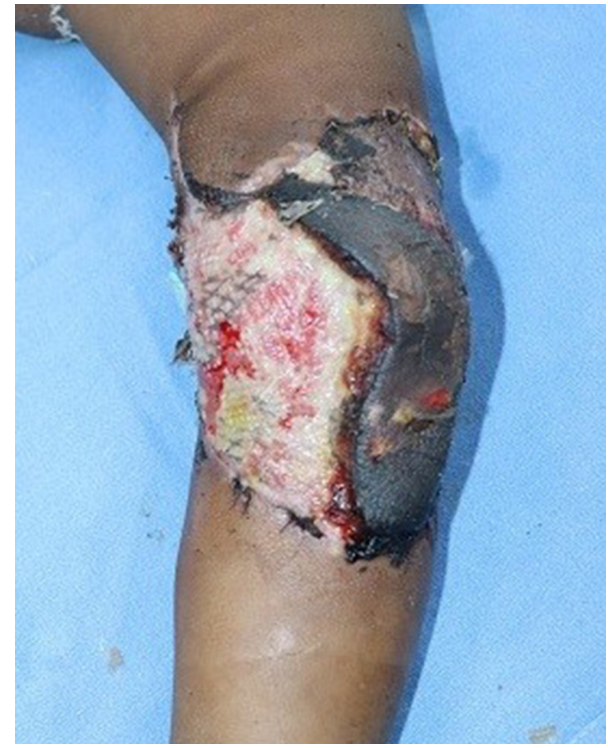

Fig. 6. Necrosis of the skin over the muscle.

was required (Fig. 5). The patient was shifted to PICU again and the flap was monitored every 2 hours from that point on for the following 5 days. An input-output chart was maintained and the baby was kept well hydrated. The loose stools resolved in 2 days. She was started on low molecular weight heparin (LMWH; enoxaparin) at a dose of $12 \mathrm{mg}$ twice daily

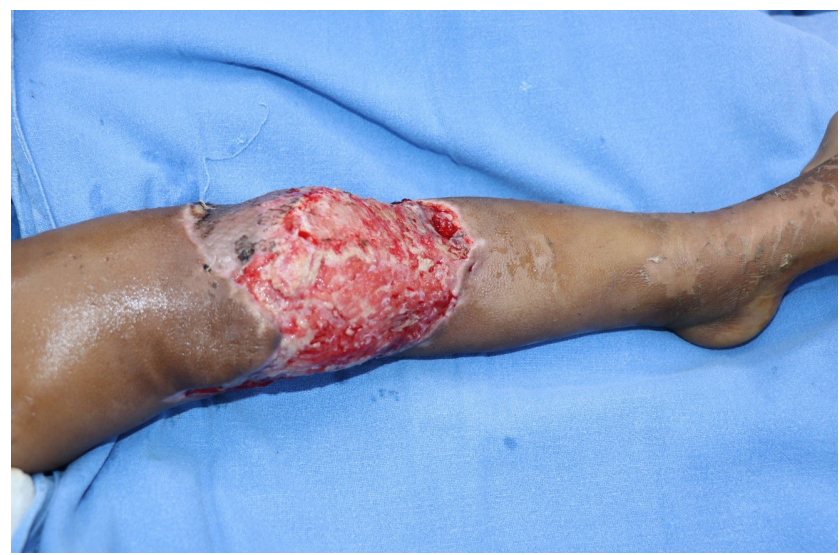

Fig. 7. Debrided skin with healthy underlying muscle.

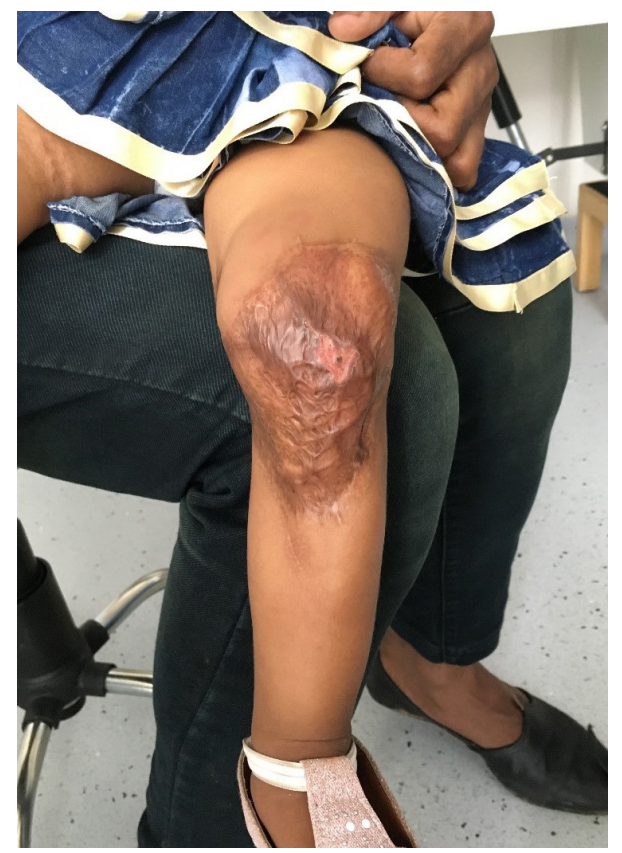

Fig. 8. Healed wound at 2 months.

right from the time of re-exploration in the operating room. Although the muscle remained healthy, the color of the skin paddle had deteriorated (Fig. 6). Five days after re-exploration, the patient underwent debridement of the skin paddle, and a skin graft for the raw area was used. The skin graft on medial side of the skin paddle was also lost while the graft on the lateral aspect of skin paddle remained adhered to the muscle (Fig. 7). The muscle underlying the skin paddle was viable.

The patient had an uneventful recovery since then (Fig. 8). At 4 months after final reconstruction her flap has settled well and she is walking without support (Fig. 9, Supplementary Video clip 1). 


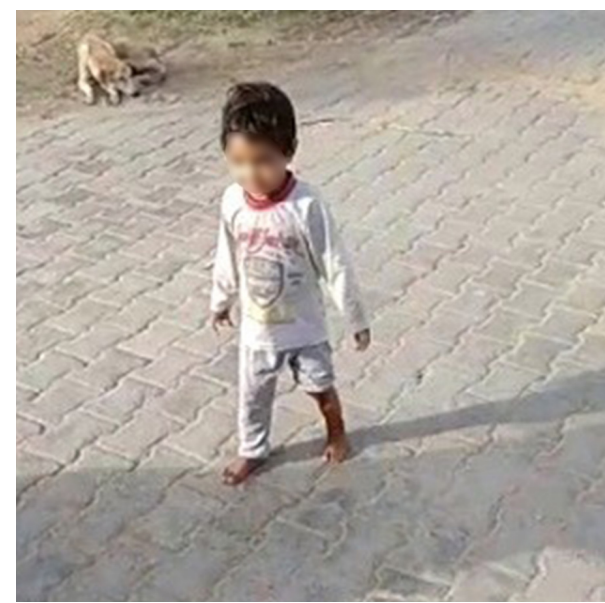

Fig. 9. Patient walking without support.

\section{Discussion}

Free tissue transfers started in the early 1970s, heralding a new era in reconstructive surgery. Soon microsurgery was introduced for reconstruction in pediatric age groups as well, when Ohmori et al. [3] in 1975 reported use of groin flaps for reconstruction in children.

The surgeons initially used muscle flaps for the pediatric group as these flaps have relatively larger vessels and they have more consistent anatomy, involving relatively straightforward dissection [4]. Van Landuyt et al. [5] showed that the size of vessels in children is larger than in adults in relation to their body size. There were also reported cases of free tissue transfer in children as young as 7 weeks old [6]. Yucel et al. [2] demonstrated that vessels in older children were thicker and more elastic, much like those of adults, as compared to younger patients whose vessels were translucent and had gelatinous consistency.

The challenges in anastomosis of smaller size vessels were eventually overcome with the gains in experience in microsurgery, and advances in operative microscopes, instruments and surgical techniques worldwide. The success rates of microsurgical free tissue transfer are equal to or better than those of the adult populations, as demonstrated by Yazar et al. (98.6\%) [7]. This may also be attributed to few or no comorbid conditions like smoking, diabetes, hypertension, atherosclerosis and drug abuse in the pediatric population. Parry even found out that the incidence of vasospasm in the pediatric age group is lower, as muscularis layer of the vessels is underdeveloped [8].

All the flaps commonly used for free tissue transfer in adults are used in children as well. Latissimus dorsi is still the most commonly used free flap in the pediatric age group, though the latest trend favors perforator flaps.

For this patient, we chose the latissimus dorsi flap because the defect area was large, and also because dissection of the latissimus dorsi is more predictable. For anastomosis, we decided on the descending genicular artery as it is one of the most common recipient vessels for flaps around the knee [9].

In retrospect, the most plausible explanation for the discolored and inadequately perfused flap would be an episode of vasospasm due to dehydration by loose stools. The spasm was relieved as hydration was reestablished, dressings loosened and core body temperature improved. Though skin flaps typically tolerate ischemia better than muscles, in our case, the skin paddle over the muscle necrosed while the underlying muscle was healthy. This might owe to a thromboembolic phenomenon (during the time of vasospasm) with embolus blocking the perforators supplying the skin. Anticoagulation is not a routine practice in our center. It is used only when re-exploring flaps. In consideration of the $6.8 \%$ incidence of thrombosis in pediatric free flaps [7], we kept the baby on LMWH for 2 weeks.

Vasospasm in children, although rare, can happen as in our case and it should always be considered a possibility whenever the perfusion in the flap seems less than adequate. The intensive postoperative monitoring should continue for 5-7 days, because unlike adults, children have poor reserves and are more likely to develop complications. We therefore suggest that free tissue transfer for younger children should be performed at specialized centers with much microsurgical experience and a good PICU. We hope these recommendations will contribute to more successes in reconstruction, as the challenges we encountered in this case are most likely to arise for others as well.

In conclusion, microsurgical free tissue transfer in younger children shows good success rates. These surgeries should be done in specialized microsurgical centers with a good PICU.

\section{Conflict of interest}

No potential conflicts of interest relevant to this article are reported.

\section{ORCID iDs}

Hardeep Singh ～https://orcid.org/0000-0001-7929-8804 Rakesh Kumar Khazanchi

https://orcid.org/0000-0002-7417-8720 
Sanjay Mahendru

https://orcid.org/0000-0001-7604-8391

Ankit Jain

https://orcid.org/0000-0002-6068-0146

\section{Supplementary material}

Video clip 1. Patient walking without support and without contracture. To refrain from unnecessarily exposing the patient's personal identity, the video clip has been limited to the posterior view.

Supplemental data can be found at: https://doi.org/10.22467/ jwmr.2020.01081.v1

\section{References}

1. Hallock GG. Efficacy of free flaps for pediatric trauma patients in an adult trauma center. J Reconstr Microsurg 1995; 11:169-74

2. Yucel A, Aydin Y, Yazar S, et al. Elective free-tissue transfer in pediatric patients. J Reconstr Microsurg 2001;17:27-36.

3. Ohmori K, Harii K, Sekiguchi J, et al. The youngest free groin flap yet? Br J Plast Surg 1977;30:273-6.
4. Wechselberger G, Radauer W, Schimpl G, et al. Lower limb salvage in a 7-month-old infant using free tissue transfer. J Pediatr Surg 2011;46:1852-4.

5. Van Landuyt K, Hamdi M, Blondeel P, et al. Free perforator flaps in children. Plast Reconstr Surg 2005;116:159-69.

6. Germann G, Waag KL, Selle B, et al. Extremity salvage with a free musculocutaneous latissimus dorsi flap and free tendon transfer after resection of a large congenital fibro sarcoma in a 15-week-old infant: a case report. Microsurgery 2006;26:429-31.

7. Yazar S, Wei FC, Cheng MH, et al. Safety and reliability of microsurgical free tissue transfers in paediatric head and neck reconstruction: a report of 72 cases. J Plast Reconstr Aesthet Surg 2008;61:767-71.

8. Parry SW, Toth BA, Elliott LF. Microvascular free-tissue transfer in children. Plast Reconstr Surg 1988;81:838-40.

9. Venkatramani H, Sabapathy SR, Nayak S. Free-flap cover of complex defects around the knee using the descending genicular artery as the recipient pedicle. J Plast Reconstr Aesthet Surg 2014;67:93-8. 